Trauma Berufskrankh 2010 • 12[Suppl 3]:316-322 DOI 10.1007/s10039-009-1585-z

Online publiziert: 10. Januar 2010

(c) Springer-Verlag 2010

P.P. Schmittenbecher

Kinderchirurgische Klinik, Städtisches Klinikum Karlsruhe

\title{
Diagnostik der Frakturen im Kindesalter
}

- die Umstände des Unfalls (Ort, Geschwindigkeit, Dynamik),

- den Erstbefund vor Ort (Perforation, Luxation) und ggf.

- dort bereits ergriffene Maßnahmen (Reposition, Medikation).

Auch in der Kürze der Zeit im Rahmen der Erstversorgung muss sich der behandelnde Arzt Gewissheit darüber verschaffen, ob der berichtete Unfall dem Verletzungsmuster entspricht, da das Gegenteil auf eine Kindesmisshandlung hinweist.

Impfstatus (Tetanus), generelle Skeletterkrankungen (Osteogenesis imperfecta, Morbus Ollier) oder sonstige begünstigende Erkrankungen (Spastik, Osteoporose) sind im Kindesalter wichtig und zu erfragen.
Die klinische Untersuchung umfasst neben der Inspektion die vorsichtige Prüfung von Durchblutung, Motorik und Sensibilität. Bei Kleinkindern stellt dies eine besondere Herausforderung dar. Der Erstbefund muss ggf. als unvollständig dokumentiert und im Verlauf ergänzt werden. Im Vordergrund steht eine Orientierung über die Möglichkeit neurovaskulärer Begleitverletzungen, die bei der geringen Zahl offener Frakturen im Kindesalter in aller Regel „gedeckt" sind. Im Fokus steht hier die suprakondyläre Humerusfraktur mit begleitender Medianusparese. Schmerzhafte Manipulationen sind in dieser Phase zu vermeiden. Dennoch sollte eine für die nachfolgende bildgebende Diagnostik wichtige Eingrenzung der Verletzungsregion möglich sein.

Die Anamnese fokussiert beim unfallverletzten Kind auf:

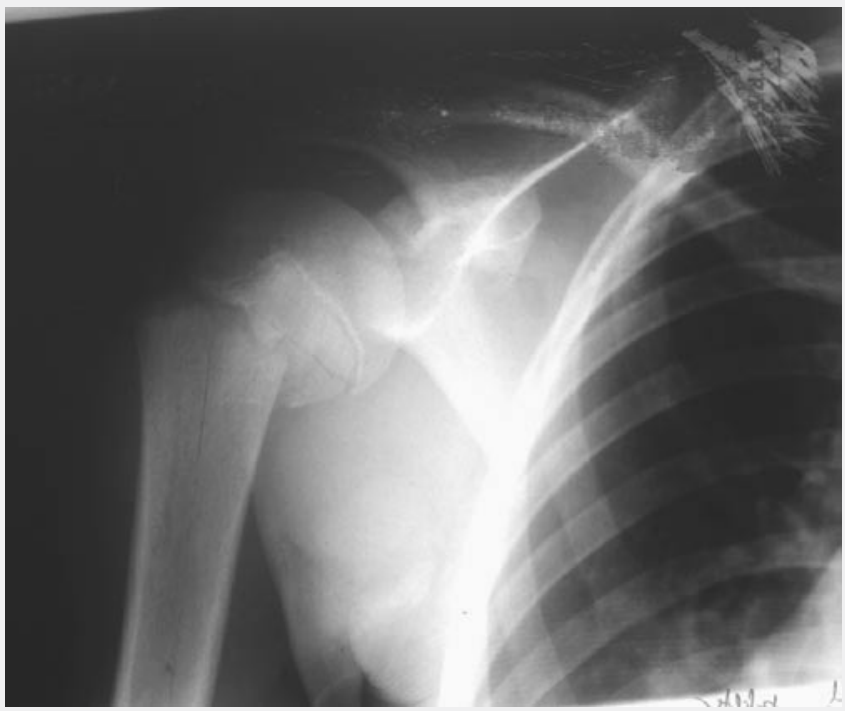

Abb. $2 \Delta$ Proximaler Oberarm a.-p., nicht erforderliche Mitabbildung von Schultergürtel und rechter Lunge 


\section{Konventionelle Röntgenuntersuchungen}

\section{Diagnose}

Am Anfang der bildgebenden Diagnostik steht grundsätzlich die Aufnahme in 2 definierten Ebenen im Winkel von $90^{\circ}$ zueinander, die in aller Regel die beiden angrenzenden Gelenke einschließen muss (- Abb. 1). Dabei ist eine vernünftige Eingrenzung der Region anzustreben [14]. Die Darstellung z. B. des proximalen Humerus darf nicht den gesamten Schultergürtel und weite Teile der Lunge umfassen (- Abb. 2), und bei einer klinisch nicht gut eingrenzbaren Verletzung des Oberarms ist die getrennte Abbildung von proximalem Oberarm und Ellenbogen v. a. wegen der besseren Beurteilbarkeit der fokussierten Ellenbogenaufnahme (- Abb. 3) empfehlenswert. Ist nach dem ersten Röntgenbild die therapeutische Entscheidung klar und die Einstellung der 2. Ebene sehr schmerzhaft, kann auf diese verzichtet und sie intraoperativ nachgeholt werden.

Ergänzende schräge Aufnahmen sind am Becken (Ala-Aufnahme, D Abb. 4) oder am oberen Sprunggelenk hilfreich, die dynamische Durchleuchtung ist nur in Einzelfällen und bei schwieriger Einstellung einer überlagerten Region (• Abb. 5) notwendig.

Für die Beurteilung der Aufnahmen müssen die Bildqualität ausreichend und das Gewünschte korrekt abgebildet sein (- Abb.6), anderenfalls sind Wiederholungsaufnahmen unerlässlich. Wer hierauf verzichtet, weil sie für die Therapieentscheidung doch nicht erforderlich erscheinen, hat eigentlich schon die Primäraufnahme nicht benötigt und damit die rechtfertigende Indikation nicht konsequent hinterfragt!

Für die Bildqualität ist die korrekte Positionierung des Patienten mit adäquatem Patientenschutz entsprechend der Einstellkompendien wichtig. Danach dient die gute Einblendung der gewünschten Region ebenso der Dosisreduktion wie die heute zunehmend verbreitete digitale Bildtechnik und -verarbeitung sowie eine individuelle Dosisanpassung. Zusammen mit den Strahlenfiltern kann so eine Dosisreduktion von $90 \%$ erreicht werden [1, $9,10,15,16]$. Die digitale Bildverarbeitung ermöglicht zudem eine Nachbearbeitung mit einer zusätzlichen Visualisation „verborgener" Aspekte durch die Veränderung von Helligkeit und Kontrast.

Die Bildverteilung ist digital ebenfalls vereinfacht, sodass dem behandelnden Arzt das Bild schnell am Bildschirm zur Beurteilung zur Verfügung steht. Die Befundung soll dabei auf einem intensiven "Lesen“ des Röntgenbildes basieren und muss z. B. die aktive Suche nach Zeichen der Instabilität beinhalten (- Abb. 7).

Eine Aufnahme der Gegenseite ist in der Akutdiagnostik frischer Verletzungen nicht hilfreich und stellt eine entbehrliche Strahlenbelastung einer nicht verletzten Körperregion dar! Die absolut vergleichbare Einstellung z. B. des verletzten und unverletzten Ellenbogengelenks bei einem Kleinkind mit frischer, schmerzhafter Ellenbogenverletzung ist unmöglich und damit auch diagnostisch nicht hilfreich. Die Identifikation von Verletzungen, die dem Arzt unbekannt sind und deshalb nicht identifiziert werden, ist auch im Seitenvergleich nicht möglich. So ist es bei der in 1 Abb. 8 dargestellten seitenvergleichenden Aufnahme des distalen $\mathrm{Hu}$ merus unmöglich, zu unterscheiden, ob rechts etwas fehlt oder links etwas zu viel vorhanden ist. Hier ist ggf. ein entsprechendes Standardwerk wie der „Röntgenatlas der Normvarianten“"von Keats [5] zu Rate zu ziehen.

\section{Kontrolluntersuchungen}

Verlaufskontrollen sollen gezielt eingesetzt werden. Sie dienen

- der Dokumentation nach jeder Manipulation,

- der Stellungskontrolle konservativ behandelter Frakturen nach 1 Woche mit der Frage der Re- oder sekundären Dislokation und

- der gipsfreien Konsolidierungskontrolle entsprechend der angenommenen Heilungszeit des Bruchs [6].

Die Überlegung weiterer Stellungskontrollen nach 1 Woche Ruhigstellung zeigt den Zweifel an der Stabilität der Therapiemaßnahme auf und sollte konkret über die Indikation zur Osteosynthese nachdenken lassen. Wöchentliche Stellungskontrollen konservativ behandelter Frakturen sind obsolet!
Trauma Berufskrankh 2010 - 12[Suppl 3]:

316-322

DOI 10.1007/s10039-009-1585-z

๑) Springer-Verlag 2010

\section{P.P. Schmittenbecher \\ Diagnostik der Frakturen im Kindesalter}

\section{Zusammenfassung}

Die Diagnostik der Frakturen im Kindesalter basiert auf dem konventionellen Röntgenbild. Schnittbildverfahren erfolgen ergänzend mit einer gezielten Fragestellung und fokussieren auf zusätzliche Informationen. Die Sonographie hat sich noch nicht als Standardverfahren durchgesetzt.

\section{Schlüsselwörter}

Frakturdiagnostik · Kindesalter $\cdot$ Röntgenbild . Schnittbildverfahren $\cdot$ Sonographie

\section{Diagnosis of fractures in children}

\section{Abstract}

The diagnosis of fractures in children is based on conventional $\mathrm{X}$-rays. Computed tomography or magnetic resonance imaging scans are done as supplementation for a specific question and with a focus on additional information. Ultrasound is still not established as a standard procedure.

\section{Keywords}

Diagnosis of fractures - Childhood · X-rays · CT or MRI scans · Ultrasound 


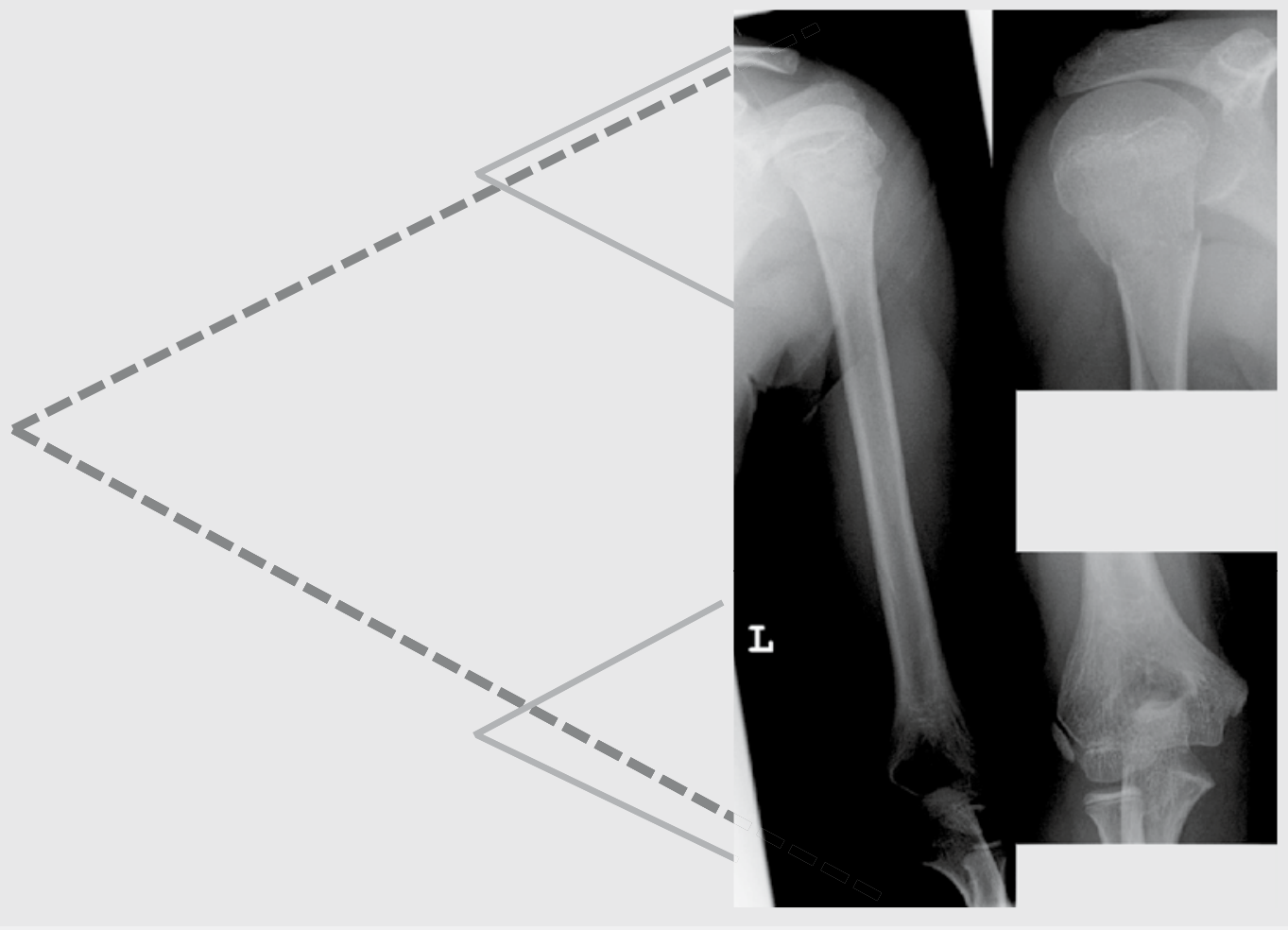

Abb. 34 Aufnahme des gesamten Oberarmes (Mitte): weniger aussagekräftig als fokussierte Darstellung von proximalem Oberarm und Ellenbogen (rechts)

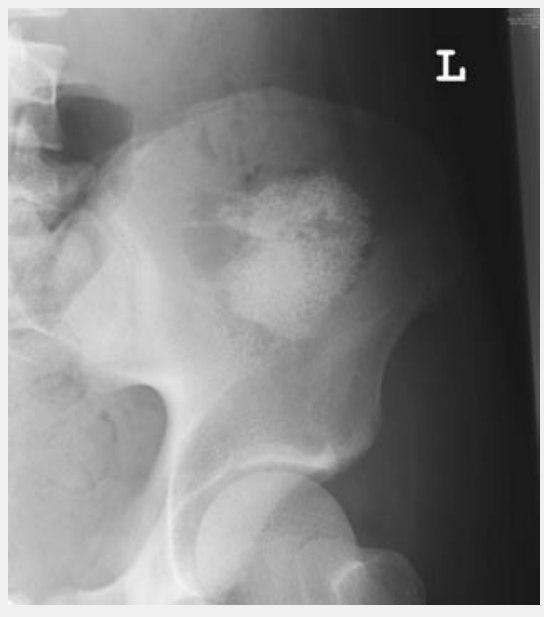

Abb. $4 \Delta$ Schräge (so genannte Ala-)Aufnahme des Beckens zur besseren Darstellung einer aufgefüllten juvenilen Knochenzyste im Verlauf
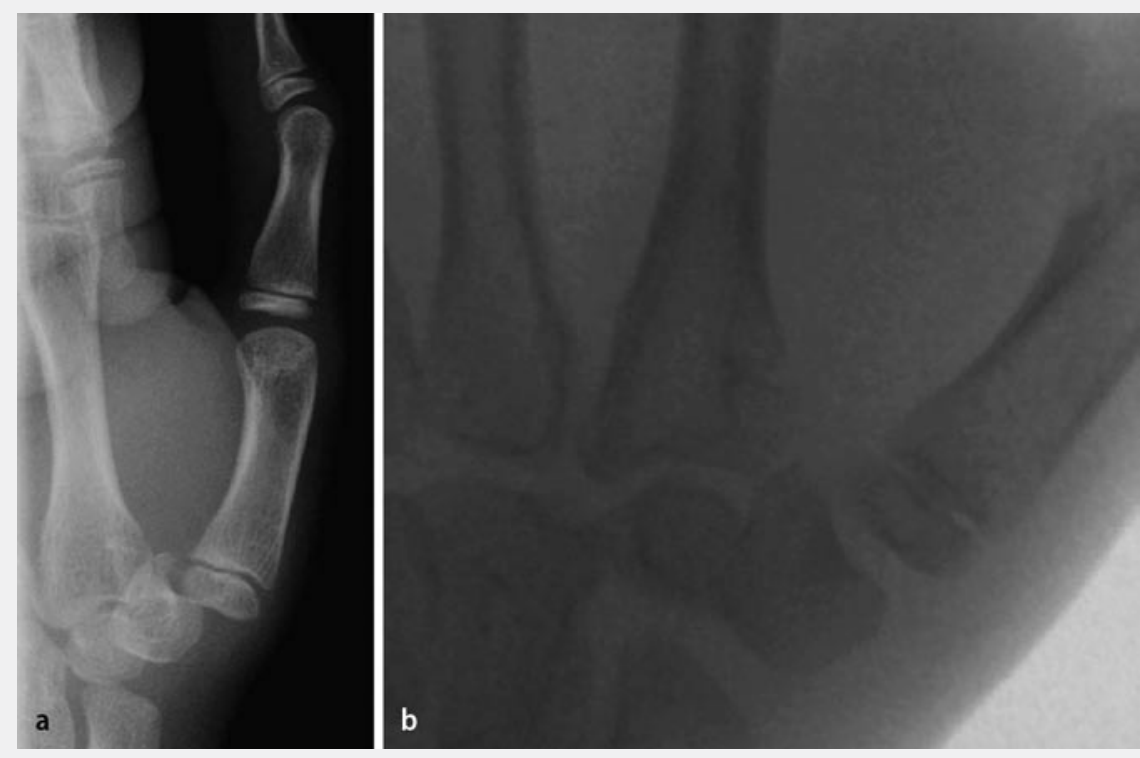

Abb. $5 \Delta$ Unklare Überlagerung der Basis des MC2 (Metacarpale 2) mit dem Trapezium (a) mit besserer Beurteilbarkeit in der Durchleuchtung (b)

\section{Ultraschall}

Er ist in der Kindertraumatologie noch nicht routinemäßig etabliert, obwohl er

- in der Diagnostik geburtstraumatischer Frakturen [4],

- bei der Beurteilung der Condylusradialis-Fraktur [13],

- in der Darstellung subperiostaler distaler Unterarmfrakturen [8] und
- bei bestimmten Verlaufs- und Konsolidierungskontrollen

eingesetzt wird. Dabei müssen zum einen eine entsprechende Erfahrung und Übung in der Beurteilung der Skelettsonographie vorhanden sein, zum anderen ist die Kooperation des Patienten zur exakten und reproduzierbaren sonographischen Ein- 
Abb. 6 - Vermeintlich unauffälliges Röntgenbild bei zu stark eingeblendeter Aufnahme (a); sekundäre Darstellung einer konsolidierten Fraktur (b)
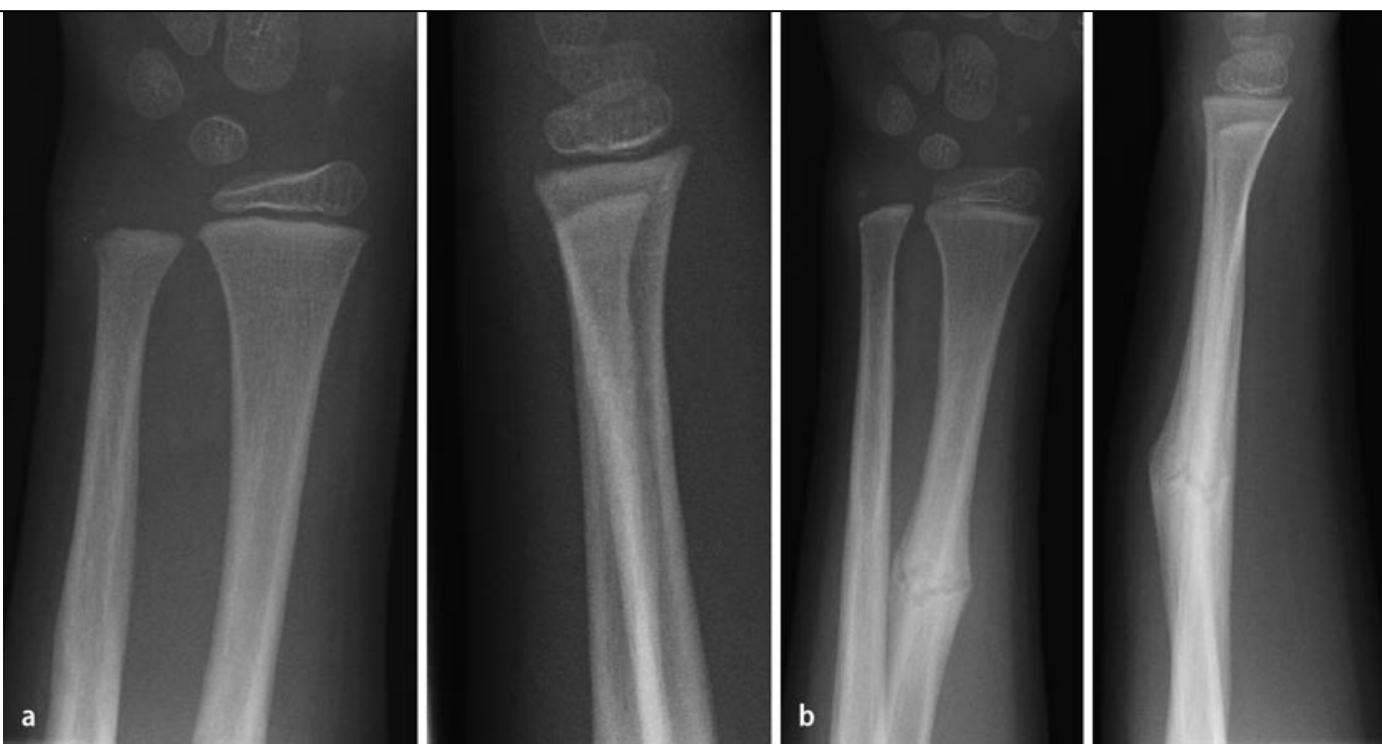

stellung der verletzten Skelettregion erforderlich.

\section{Computertomographie}

Ihr Wert steht in der Beurteilung von Wirbelsäulen- oder Beckenverletzungen ebenso außer Frage wie in der Polytraumadiagnostik. In der Evaluation der Extremitätenfrakturen hat sie v. a. am oberen Sprunggelenk, aber auch bei anderen unklaren gelenknahen Verletzungen ihre Bedeutung $[11,12]$. Wenn immer im konventionellen Röntgenbild einer gelenknahen Fraktur unklare oder verdächtig erscheinende Aspekte beobachtet werden oder wenn Bild und Klinik im Schweregrad der Verletzung deutlich auseinanderweichen, kann die CT zur diagnostischen Klärung beitragen.

Am oberen Sprunggelenk ist die CT oft hilfreich, den dreidimensionalen Verlauf der Fraktur und damit die Frakturdynamik bei den Übergangs- und einzelnen Innenknöchelfrakturen klarer darzustellen und damit das operative Vorgehen und die genaue Schraubenplatzierung zu vereinfachen (• Abb. 9, [3]). Hierzu ist jedoch keine $3 \mathrm{D}$-Rekonstruktion erforderlich, die genau diesen Aspekt oft nicht zeigen kann, sondern die 2D-Darstellung ist effizienter.

Die Untersuchung soll dabei nicht Unbekanntes oder Unerkanntes zeigen (- Abb. 10), sondern Unklares differenzieren (『 Abb. 11).

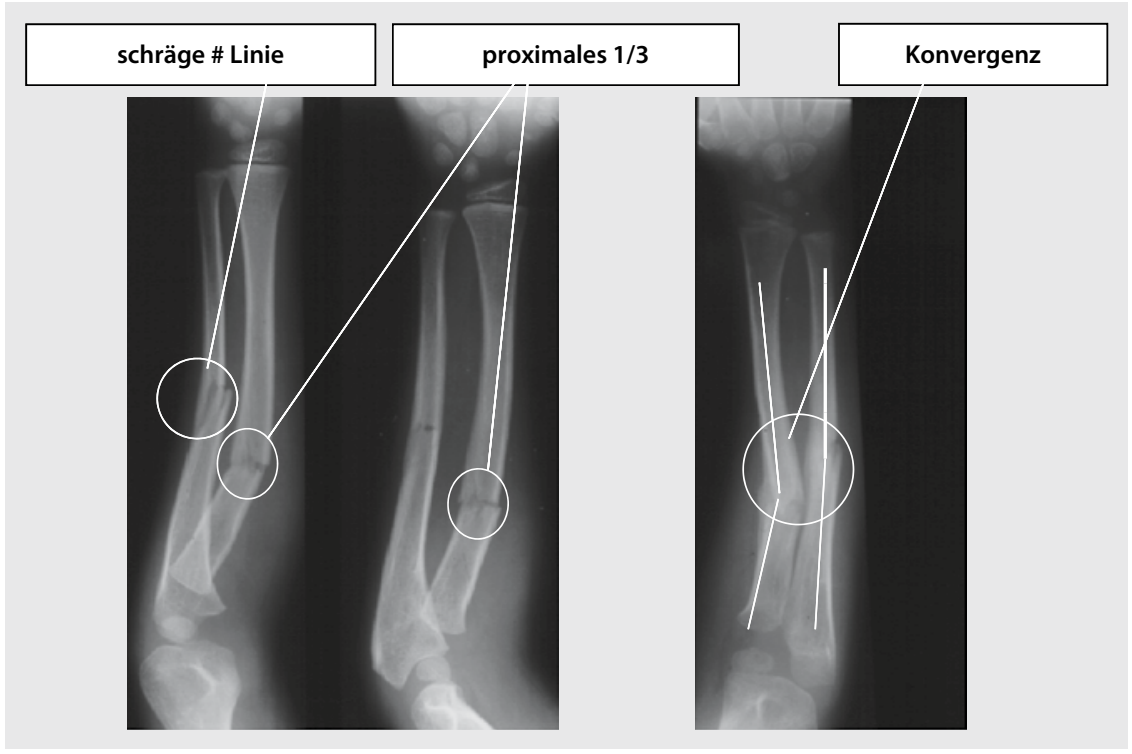

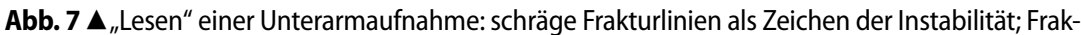
turen im proximalen Drittel und konvergierende Frakturlinien als Zeichen drohender Funktionsstörung

\section{Magnetresonanztomographie}

Ihre Bedeutung in der Diagnostik von Frakturen im Kindesalter wird in der Arbeit von Schneidmüller et al. [7] in diesem Heft diskutiert.

\section{Diskussion}

Die Diagnostik der Frakturen im Kindesalter basiert unverändert auf der Bildgebung im konventionellen Röntgenbild. Nach Anamnese und klinischer Untersuchung ist eine gezielte und fokussierte konventionelle Röntgenuntersuchung zu veranlassen $[2,14]$. Hierbei muss eine gute Bildqualität gesichert sein [1]. Daneben sind aber v. a. die kritische Bildbewertung („Lesen“ des Bildes) und Befundung erforderlich, da sie die entscheidende therapeutische Weichenstellung induzieren. Grundlage der Bewertung ist die Kenntnis der altersspezifischen Anatomie und der Dynamik der Skelettentwicklung. Der Zeitpunkt des Erscheinens, die sich ändernde Morphologie und das ortsspezifische Verknöchern der Knochenkerne müssen demjenigen, der Kindertraumatologie und -radiologie betreibt, geläufig sein. 


\section{Frakturen bei Kindern und Jugendlichen}
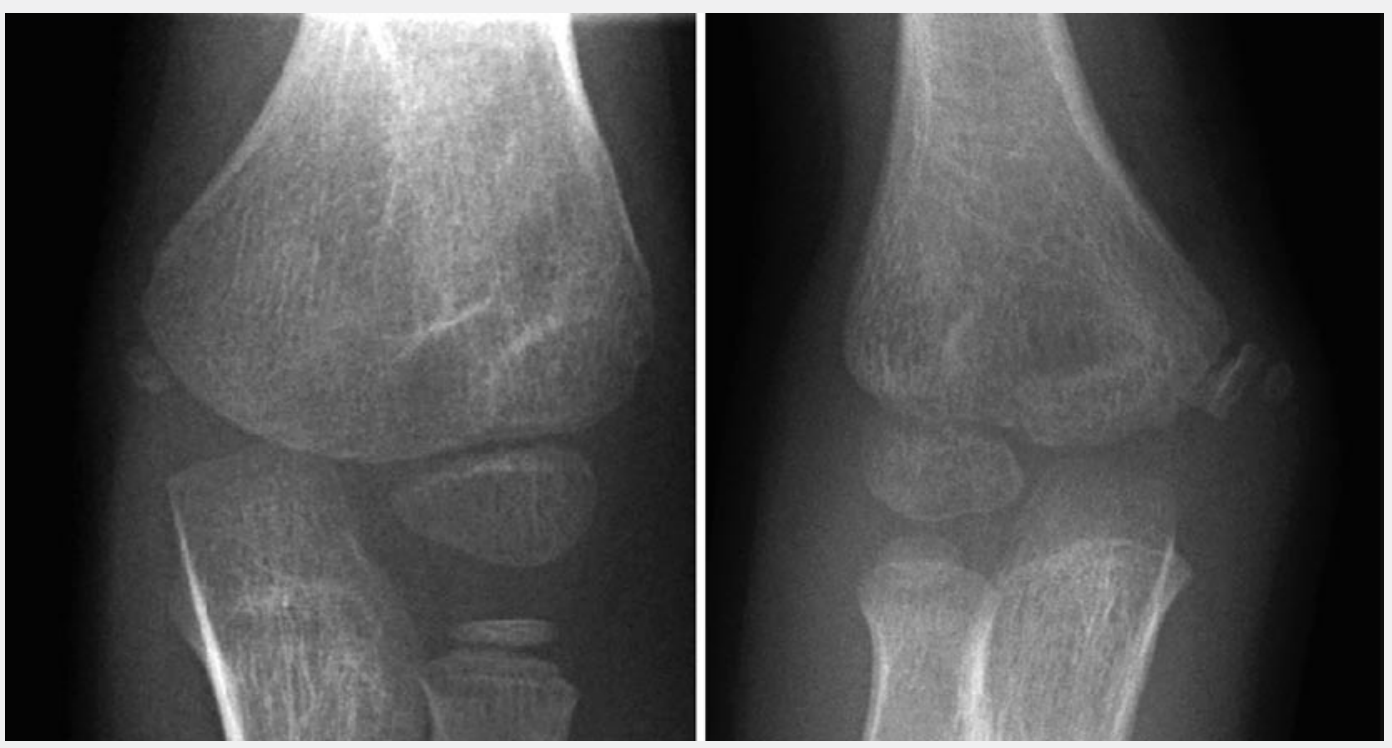

Abb. $8<$ Seitenvergleichende Aufnahmen des distalen Humerus mit ungewöhnlicher Abrissfraktur des Condylus ulnaris links
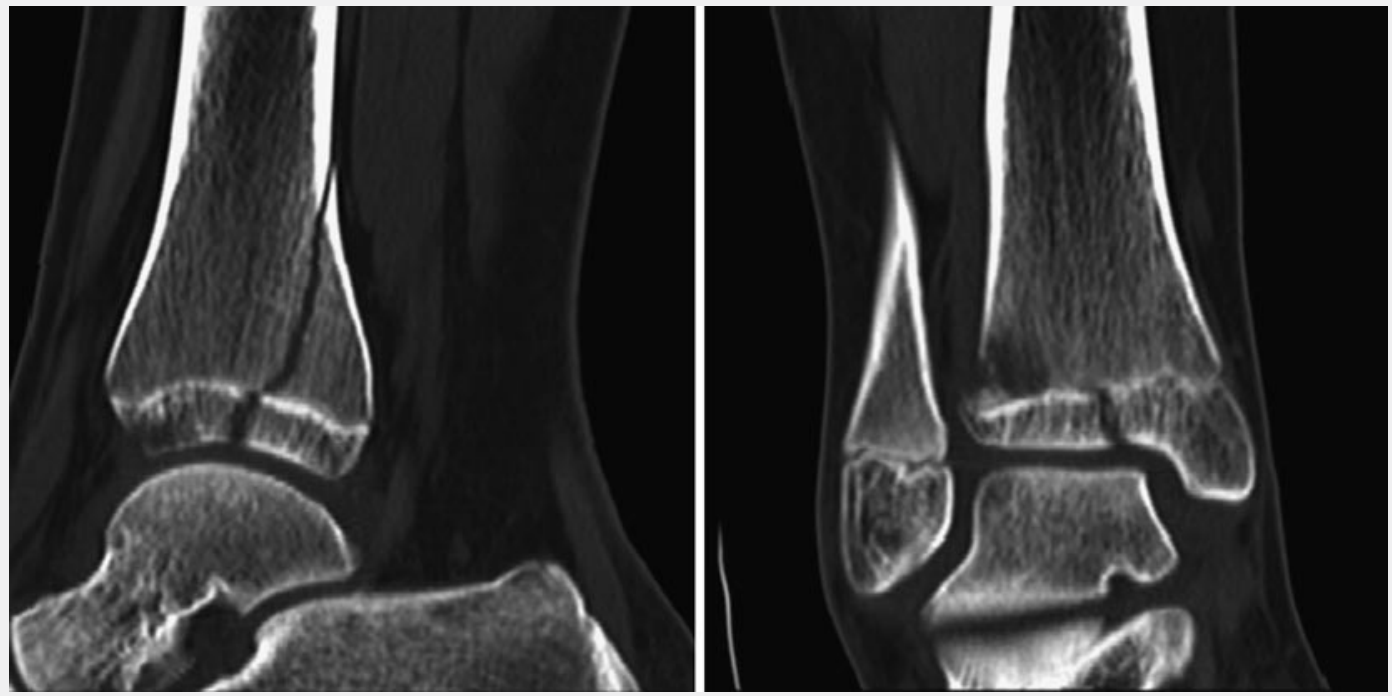

Abb. $94 \mathrm{CT}$ einer Übergangsfraktur Triplane-Typ I
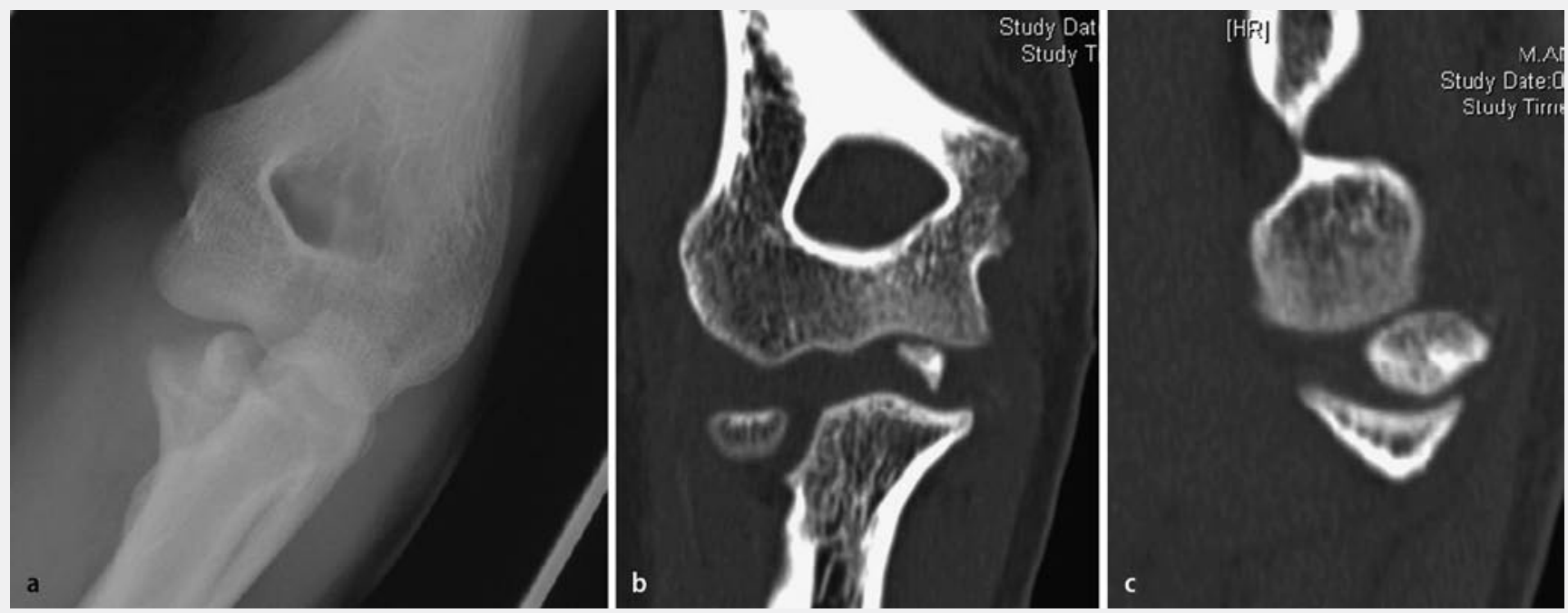

Abb. $10 \Delta$ Konventionelles Röntgenbild mit eindeutigem Epicondylus-ulnaris-Abriss (a); überflüssige CT-Untersuchung mit identischem Befund $(\mathbf{b}, \mathbf{c})$ 
Verlaufskontrollen werden oft routinemäßig veranlasst und folgen überholten Algorithmen. Sie sollen jedoch begrenzt eingesetzt werden und müssen nicht das Remodelling begleiten [6]. Aus jedem veranlassten Bild muss theoretisch eine Konsequenz erwachsen können. Ist diese aufgrund Frakturlokalisation, Frakturmorphologie oder Patientenalter von vorneherein nicht gegeben, ist auch die Aufnahme entbehrlich.

In der täglichen Praxis wird immer noch die Röntgenaufnahme der gesunden Gegenseite veranlasst, um im Seitenvergleich die individuelle Norm, aber auch das Pathologische zu identifizieren. Die gelenknahen Frakturen stehen hierbei im Fokus, da die Differenzialdiagnose „Fraktur" vs. „Fuge“ Probleme bereitet. Hier gilt natürlich das oben hinsichtlich der Anatomie Angeführte in gleicher Weise. Zudem ist jedoch in Betracht zu ziehen, dass ein Vergleich der beiden Aufnahmen nur dann zielführend sein könnte, wenn diese in der exakt gleichen Position angefertigt werden würden, da sich durch geringe Rotationsdifferenzen die epiphysären Kerne völlig unterschiedlich darstellen können. Die Vergleichsaufnahme ist deshalb in der Akutdiagnostik von Frakturen obsolet und kann nur noch wirtschaftlichen Zwecken dienen.

Ergänzende Bildgebung mittels Schnittbildverfahren soll mit einer gezielten Fragestellung erfolgen und auf zusätzliche Informationen fokussieren. Die Flut überflüssiger CT- und MRT-Untersuchungen muss eingedämmt werden. Auch hier ist kritisch anzumerken, dass das Schnittbildverfahren nicht die Aufgabe haben kann, die unzureichende Erfahrung des behandelnden Arztes mit den Besonderheiten des Skeletts im Kindesalter zu kompensieren. Die Frage muss immer sein, welche konkrete Befunddifferenzierung und welche therapeutische Konsequenz aus der Zusatzuntersuchung gezogen werden sollen. Deshalb darf die Fragestellung nicht lauten: „Liegt eine Fraktur vor oder nicht?".

Ist die Fraktur auf dem konventionellen Bild nicht zu erkennen, kann sie schlechterdings auch nicht disloziert sein. Damit ergibt sich keine Operationsindikation. Die Frage der Ruhigstellung ist aus der Klinik, ihre Dauer aus dem klinischen
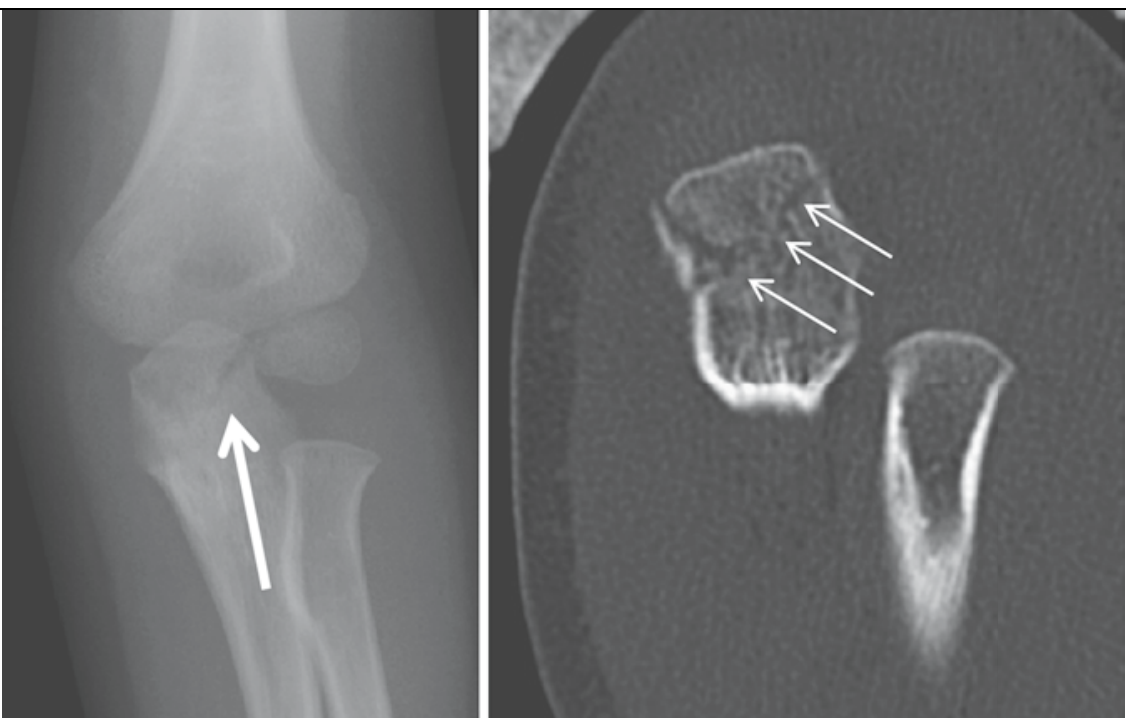

Abb. 11 \ Diagonale Olekranonfraktur durch die Gelenkfläche bei Kleinkind, Frage der Operationsindikation wegen möglicher Gelenkstufe (a); CT: keine relevante Stufe oder Dehiszenz der Gelenkfläche (b)

Verlauf zu beantworten. Nur wenn die Diagnose der Fraktur feststeht, das Röntgenbild als Summationsbild aber die Frage der Dislokation im Raum nicht beantworten kann und damit die Notwendigkeit einer Operation ungeklärt bleibt, ist die CT-Untersuchung sinnvoll. Dabei kann die $2 \mathrm{D}$-Rekonstruktion in der fraglichen Ebene sehr viel mehr Antworten geben als die moderne $3 \mathrm{D}$-Rekonstruktion, die letztendlich nur eine Außenbetrachtung des Knochens aus verschiedenen Richtungen ermöglicht.

Weiterentwicklungen sind v. a. für die Ultraschallanwendung zu erwarten. Es mehren sich die Berichte, dass subperiostale Frakturen im Ultraschall diagnostiziert und im Verlauf kontrolliert werden können [8]. Aber auch in der Beurteilung der Gelenkbeteiligung von Frakturen im Kleinkindesalter findet das Verfahren Anwendung, um Spalten, Stufen oder Verschiebungen der chondralen, im Röntgenbild nicht sichtbaren artikulären Abschnitte aufzudecken und die sachgerechte Therapieentscheidung möglich zu machen [13]. Darüber hinaus ist der Ultraschall bei der Diagnostik geburtstraumatischer gelenknaher Verletzungen unentbehrlich, da die bei der Geburt an vielen Stellen noch fehlenden epiphysären Knochenkerne die konventionell-radiologische Diagnose einer Epiphysenlösung unmöglich machen [4].

Kindertraumatologe und Kinderradiologe müssen zusammenarbeiten, um durch einen effizienten und Strahlen sparenden Einsatz der konventionellen Bildgebung in den meisten Fällen von Skelettverletzungen im Kindesalter die Basis für eine gute Diagnose und Therapieentscheidung zu legen und aus der kritischen Analyse des konventionellen Bildes die Indikation zur Schnittbilddiagnostik zu stellen.

\section{Korrespondenzadresse}

Prof. Dr. P.P. Schmittenbecher

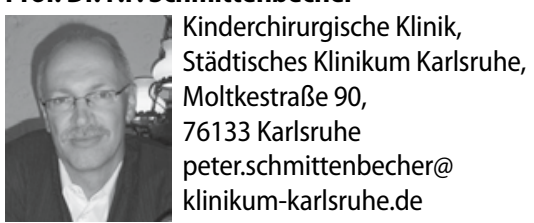

Interessenkonflikt. Der korrespondierende Autor gibt an, dass kein Interessenkonflikt besteht.

\section{Literatur}

1. Cook JV, Kyriou JC, Pettet A (2001) Key factors in the optimization of paediatric X-ray practice. $\mathrm{Br} J$ Radiol 74:1032-1040

2. Flocken W, Bassir C, Freyschmidt J (2006) Diagnostische Besonderheiten. In: Weinberg A-M, Tscherne $H$ (Hrsg) Unfallchirurgie im Kindesalter, Bd 1. Springer, Berlin Heidelberg New York, S 57-77

3. Horn BD, Crisci K, Krug M (2001) Radiologic evaluation of juvenile Tillaux fractures of the distal tibia. J Pediatr Orthop 21:162-164

4. Jones GP, Seguin J, Shiels WE (2003) Salter-Harris II fracture of the proximal humerus in a preterm infant. Am J Perinatol 20:249-253

5. Keats TE (1990) Röntgenatlas der Normvarianten. Fischer, Stuttgart 
6. Schmittenbecher PP, Menzel C (2005) Frakturen im Kindesalter. In: Weigel B, Nerlich M (Hrsg) Praxisbuch Unfallchirurgie, Bd 2. Springer, Berlin Heidelberg New York, S 867-930

7. Schneidmüller D (2010) Magnetresonanztomographie bei kindlichen Gelenkverletzungen - therapeutische Relevanz. Trauma Berufskrankh im Druck

8. Simanovsky N, Lamdam R, Hiller N, Simanovsky N (2009) Sonographic detection of radiographically occult fractures in pediatric ankle and wrist injuries. J Pediatr Orthop 29:142-145

9. Strauss KJ (2006) Pediatric interventional radiography equipment: safety considerations. Pediatr Radiol [Suppl 2] 36:126-135

10. Strauss KJ, Kaste SC (2006) ALARA in pediatric interventional and fluoroscopic imaging: striving to keep radiation doses as low as possible during fluoroscopy of pediatric patients - a white paper executive summary. J Am Coll Radiol 3:686-688

11. Täger G, Podleska LE, Ruchholtz S et al (2007) Epiphysennahe Frakturen bei Kindern; Teil I: Obere Extremität. Unfallchirurg 110:867-883

12. Täger G, Hussmann B, Lendemans S, Nast-Kolb D (2009) Epiphysäre und epiphysennahe Frakturen bei Kindern; Teil II: Untere Extremität. Unfallchirurg 112:185-201

13. Vocke-Hell AK, Schmid A (2001) Sonographic differentiation of stable and unstable lateral condyle fractures of the humerus in children. J Pediatr Orthop B 10:138-141

14. Vogl ThJ, Wetter A, Schneidmüller D (2006) Radiologische Diagnostik. In: Marzi I (Hrsg) Kindertraumatologie. Steinkopff, Darmstadt, S 39-49

15. Willis CE (2004) Strategies for dose reduction in ordinary radiographic examinations using $C R$ and DR. Pediatr Radiol [Suppl 3] 34:196-200

16. Willis CE, Slovis TL (2004) The ALARA concept in pediatric $C R$ and $D R$ : dose reduction in pediatric radiographic exams - a white paper conference executive summary. Pediatr Radiol [Suppl 3] 34: S162-S164 